\title{
AFFECTIVE VARIABLES IN SECOND LANGUAGE ACQUISITION
}

\author{
H. Douglas Brown1 \\ University of Michigan
}

\begin{abstract}
Affective variables have not been adequately investigated in the study of second language acquisition. Imitation, egoism, and inhibition are three egocentric factors which have been treated only lightly in previous research. Three social variables, empathy, introversion/extroversion, and aggression, may be keys to understanding the social nature of second language learning. And the merging of cognition and affect in "cognitive styles," which vary within and among individuals, might account for varying degrees of success in learning a second language. Widespread interdisciplinary research in the affective domain of the psychology of language acquisition could lead to the construction of a comprehensive theory of second language acquisition as well as more effective approaches to language teaching.
\end{abstract}

Any survey of the current state of the art in language teaching leaves little doubt that the profession is undergoing a revolution. The present revolution is taking place according to a predictable twenty-five-year timetable: around 1900 the "direct" method was popular; some twenty-five years later "grammar translation" methods were taking hold; and in the 1950's the now well-established audiolingual method came into vogue. The late 1970's should usher in a new paradigm.

While the nature of the next "method" is still unknown, there is clearly a strong tendency toward interdisciplinary solutions to language teaching problems, with psychology occupying a key role, as it did with the audiolingual method. However, new schools of psychology have arisen since the 1950's, providing a different thrust, especially in the psychology of learning. The cognitive approach to human learning has important implications for both a theory of second language acquisition and more effective approaches to language teaching (Brown 1972a, 1972b). But an equally important psychological domain to explore in trying to understand the process of second language acquisition is the affective domain. We must acknowledge that while all the optimal cognitive factors may be operating in the attempted solution of a given task, the

1 The author is grateful to Professor Ronald Wardhaugh of the University of Michigan for assistance in the preparation of the manuscript. 
learner can fail because of an affective block. Ten years ago Ernest Hilgard (1963:267) warned that "purely cognitive theories of learning will be rejected unless a role is assigned to affectivity." Today there is an increasing awareness of the necessity to examine the human personality to find answers to perplexing problems in language learning. Research studies by Gardner and Lambert (1972), Lukmani (1972), Guiora et al. (1970, 1972), and others have, on a theoretical level, provided insights into the role of the affective domain in language learning. Other more practically oriented studies have demonstrated the feasibility of classroom methodology oriented to the affective side of human behavior (Savignon 1972, LaForge 1971, Begin 1971). Stevick's (1973) review of Curran (1972) provides an interesting background summary of the principles used by LaForge and Begin.

The research carried out to date is only a beginning. Much more experimentation is needed before we can adequately define a theory of second language acquisition and devise language teaching methods which are solidly grounded in both cognitive and affective principles. One of the greatest difficulties in striving for such a goal is subdividing and categorizing the factors of the affective domain. We are often guilty of using rather sweeping terms as if they were carefully defined. For example, it is easy enough to say that "culture conflict" accounts for most language learning problems, or that "motivation" is the key to success in a foreign language; but it is quite another matter to define such terms with precision. Psychologists also experience a difficulty in defining terms. Abstract concepts such as "ego," "aggression," "identification," and other common terms are difficult to define operationally. Standardized psychological tests often form an empirical definition of such concepts, but constant revisions are evidence of an ongoing struggle for validity. Nevertheless, the elusive nature of affective and cognitive concepts need not deter us from seeking answers to questions. Carefully controlled, systematic study of delimited areas of second language acquisition will ultimately lead to a greater understanding of the phenomenon and, consequently, to more effective language teaching methods.

The discussion that follows deals with a few subcategories from the affective domain that demand immediate attention from researchers. Three general affective areas will be discussed; a) egocentric factors, b) social factors, and c) cognitive style.

\section{Egocentric factors}

Imitation is one egocentric factor which needs exploration in reference to second language acquisition. Textbooks in personalitv 
development refer to a significant body of research on imitative behavior, and studies in first language acquisition have considered the role of imitation in the acquisition of language. In second language acquisition, however, very little consideration has been given to imitation. It has been questionably assumed that one can learn the patterns of a foreign language chiefly or exclusively by imitation. This assumption has little relationship to the deeper issue of the degree and direction of affective imitative behavior in human beings. Miller and Dollard's (1941) classic work on imitation integrated the concept of imitation into a behavioristic framework and presented the problem as a major issue for learning theorists. Since then Mowrer (1960) has provided valuable data on imitative learning with some major references to language. Other concepts related to imitation-identification, modeling, dependence, and even inhibition-emerge as important factors in personality development. Ausubel's (1952) study of the school performance of "satellizers" (those who tend to attract imitators) and "nonsatellizers" (usually imitators of and dependent upon superordinate figures) found important differences between the two types of individual.

We have not even begun to explore the full implications of individual differences among persons in reproducing actions, attitudes, or linguistic behavior exhibited by models of various kinds. For example, a novel understanding of "culture" could emerge from a comparative study of imitation and identification across cultures. Also, many language teaching methods depend upon both affective imitation and linguistic repetition (a form of psycho-motor imitation); how could such methods be made optimally effective? Children have been found to be rather poor linguistic imitators (McNeill 1966), attending to truth value more than to surface structure data; could it be that imitation in second language learning plays a similarly deep-seated role and that mechanical oral repetition is of little consequence?

A second factor related to the thinking and feeling human organism is the "ego." The self-knowledge, self-esteem, and selfconfidence of the language learner could have everything to do with success in learning a language. Guiora et al. (1972) proposed the notion of "language ego" to refer to the very personal, egoistic nature of second language acquisition. Any language acquisition process that results in meaningful learning for communication involves some degree of identity conflict regardless of the age and motivation of the learner. Even in cases of "instrumentally" motivated language learning, a person is forced to take on a new identity if he is to become competent in a second language. The very definition of communication implies a process of revealing 
one's self to another. Breakdowns in communication often result from a person's unwillingness to be "honest" in revealing this self. A strong language ego is thus conceivably positively correlated with success in second language learning.

One specific area of potential research on egocentric factors in second language acquisition is that of the role of "motivation" in language learning. According to Ausubel (1968), one of the key factors contributing to motivation for any task is a basic egoenhancement drive: a person diligently strives for those things that tend to build or restore self-esteem. Reports and studies on motivation (Nelson and Jakobovits 1970, Gardner and Lambert 1972) have typically dealt with intelligence, aptitude, perseverance, attitude, cognitive style, and a number of other variables, but egoenhancement has been dealt with only briefly. An unpublished study (Lederer 1973) which used some of Lambert's instruments revealed that the "self-concept" of Detroit high school students was an overwhelming indicator of success in a foreign language. More research on ego-enhancement might further define how to appeal to ego-enhancement and how to incorporate ego-enhancing variables into materials and methods of language teaching.

A third area of needed research on egocentricity is the problem of inhibition within the human organism. Guiora et al. (1970) have produced one of the few studies on inhibition in relation to second language learning. Claiming that the notion of ego boundaries is crucial to the language learning task, Guiora designed an experiment using small quantities of alcohol to induce temporary states of less than normal inhibition in an experimental group of subjects. Performance of the alcohol-induced subjects on a pronunciation test in Thai was significantly better than the performance of a control group. Guiora concluded a direct relationship existed between inhibition (a component of language ego) and pronunciation ability in a second language. There were some serious problems in his conclusion: alcohol may lower inhibitions but alcohol also tends to affect muscular tension, and the latter may have been a more important factor than the former in the superior pronunciation performance of the alcohol-induced subjects; and pronunciation may be a rather poor indicator of overall language competence. Nevertheless, Guiora has emphasized an important variable which now needs further exploration. The work of Curran (1972) and his colleagues Begin (1971) and La Forge (1971) are excellent attempts to demonstrate the notion that inhibition may be one of the key obstacles to any learning which necessitates communication or interaction with another person. Savignon's (1972) experiment in "communicative competence" also dealt with the need to reduce 
inhibitions in language classes. However, further research is needed in order to discover more precisely how to reduce inhibition in the language classroom.

Social factors

The human being is a social animal and the chief mechanism for maintaining the bonds of society is language. Each language reflects and interacts with the particular society with which the language is associated. The process of second language acquisition must; therefore, by definition, involve an interaction with a new society and a linguistic and extra-linguistic understanding of that speech community. Highly sophisticated linguistic methods of language teaching have often failed to accomplish the goal of communicativity in the learner because of the highly social nature of language. While most language teachers recognize the importance of the social aspect of language, they tend to place all socially oriented language learning problems into the single category of "culture" differentiation. Such a generalization is not justified if one considers the extremely complex nature of culture. Attention to some rather obvious yet little-researched psychological variables of social behavior might reveal some important social factors in language acquisition. Three such variables will be discussed: empathy, introversion/extroversion, and aggression. All three are strongly related to egocentric factors by definition, but they all involve one individual's relationship with other individuals.

Empathy is the process of "putting yourself into someone's else's shoes," of reaching beyond the self and understanding and feeling what another person is understanding or feeling; it is probably the major factor in the harmonious co-existence of individuals in society. Language is one of the primary means of emphathizing. Kinesic, kinesthetic, proxemic, and other paralinguistic modes of communication facilitate the process of emphathizing, and must not be overlooked. Guiora and his colleagues $(1970,1972)$ have produced the only studies dealing specifically with the relationship of empathy to second language learning. In a recent study, Guiora et al. (1972) found that a modified version of the Micro-Momentary Expression (MME) test, a test of degree of empathy, successfully predicted authenticity of pronunciation of a foreign language. They recommended adding the MME to tests of intelligence and language aptitude to produce a "powerful predictive battery" (p. 111). Further exploration is needed in order to define more carefully the relationship implied by Guiora's results.

If indeed a high degree of empathy is predictive of success in language learning, it would be invaluable to discover how one could 
capitalize on that fact in language teaching. It is one thing to claim, as does Guiora, to be able to predict success, and quite another matter to cause success by fostering empathy in the language classroom. One would need first to determine if empathy is a basic disposition acquired in early childhood and/or relatively unchangeable, or if it is something one can "learn" in the adult years. If the former is true, then certain persons might indeed be more predisposed to language learning, and teachers could take this fact into account. If the latter is true, then it would not be unreasonable to carry out research on the incorporation of empathy into language teaching methods. How effective is role playing in a language class? What kinds of drills and exercises could be devised which require a person to predict or guess another person's response? How worthwhile would it be to attempt to organize foreign language classes which operate on a high-empathy basis, as in LaForge's (1971) method, where principles of $\mathrm{T}$-group therapy are used to aid the language learning process? Other questions arise, all of which need to be carefully researched.

A second social variable that has not received much attention in language acquisition research is introversion and extroversion. It is a common belief among teachers in general, particularly in Western society, that introversion is an undesirable behavior. The outgoing, amiable, talkative personality tends to be held up as axiomatically desirable and ideal from the standpoint of mental hygiene. This "cult of extroversion" carries over into the language classroom as well. Quiet, reserved personalities are treated as "problems" and language teachers seek ways of encouraging extroversion. The syndrome is further complicated by the tendency in modern language teaching to emphasize speaking in the classroom with all too little emphasis on aural comprehension.

Educational psychologists tend to agree that a child's introversion and extroversion may be "a grossly misleading index of social adjustment" (Ausubel 1968:413). It is equally conceivable that the role of introversion and extroversion may be misinterpreted in language classes. Is it indeed true that the "proficiency" of a more introverted person is qualitatively lower than his extroverted counterpart? And do those students whom a teacher assumes to be introverted actually classify as such, if the loaded variable of culture is taken fully into account? Teachers are prone to stereotype certain people as introverted or extroverted on the basis of their cultural background. However, a careful analysis of the socio-linguistic and extra-linguistic expectations of that culture could significantly change such judgments. Several areas of potential research emerge. First, one could explore, much in the same 
style as Guiora et al.(1972), the relationship between extroversion, as measured by psychological tests, and proficiency or aptitude in second language acquisition. A relationship may hold only for speaking a foreign language, and not for aural and reading comprehension and writing. Second, the use and encouragement of histrionics in the classroom could be studied carefully: what facilitating or interfering effect does a method have which incorporates drama, pantomime, humor, and a high degree of overt personality exposure on the part of each student? A third area of research could involve an examination of kinesic factors in communication to determine both the degree to which such factors are essential to foreign language learning, and the relationship among kinesics, communicativity, and introversion and extroversion.

Aggression is a third social variable in language learning which has received only minor attention, yet one which could be an important factor in a theory of language acquisition. Studies in child development take the question of aggression quite seriously, and a description of any adult personality would be inadequate if the notion of aggression were omitted from consideration. Aggression can be defined in a number of different ways, ranging from "a sequence of behavior the goal of which. . . is injury of the person toward whom it is directed" (Dollard, Doob, Miller, Mowrer, and Sears 1939:9), to more general definitions that include reference to responses that could injure or damage if aimed at a vulnerable object (Bandura and Walters 1963:114). There is also some debate about the relationship of frustration to aggression; Freud's early theory of aggression, for example, maintained that aggression is a " primordial reaction" to frustration (Freud 1920). Nevertheless, aggressive behavior appears in every human organism, and depending upon the individual and his society, different manifestations of aggression will be observed. But aggression should not be defined only in a negative context. Aggression, even in the "injurious" sense, is perhaps a behavior necessary for survival; for example, consistent refusal to be aggressive in mere self-defense, physically and emotionally, could result in physical or mental illness and/or death.

How does the notion of aggression relate to language learning? Even the exhaustive questionnaires of Gardner and Lambert (1972) have not dealt directly with aggression. Some reference to aggression is made indirectly by LaForge (1971) in his discussion of the manifestation of hostility in his experimental language classes; he noted that the overt display, and thus eventual release, of hostility seemed to facilitate communication and to lead toward less inhibited and freer, albeit semi-grammatical, conversation. The 
potential disadvantage of thwarted aggressive instincts is intuitively understood by most teachers. However, a number of other questions related to aggression are not yet answered. To what extent does the basic aggressiveness of the individual indicate his probable success in learning a foreign language? Because of its negative connotations, aggressive behavior may be considered undesirable; yet there is ample evidence of the facilitating effects of aggression. Aggression could be a central factor determining "motivation," and foreign language teaching methods might indeed capitalize on aggressive behavior. An experimental program in reading being devised at the English Language Institute of the University of Michigan is attempting to use the natural tendency to achieve a release from frustration as a motivation to comprehend reading passages about frustrating problems with which a student can easily identify. Aggressive determination to complete the lesson successfully will bring resolution. How can teachers successfully encourage students to "let it all hang out" to an optimal degree?

\section{Cognitive style}

A third major category of affective behavior that warrants attention is the notion of "cognitive style." It is difficult to argue that cognitive style is a strictly affective factor; it is more a combination of affect and cognition. As used here, it refers generally to self-consistent and enduring individual differences in cognitive organization and functioning. For example, an algebra "story" problem, typical of high school math classes, may be solved by a number of different but equally effective strategies, depending upon the individual's cognitive style. The affective domain is relevant because variations in cognitive style appear to covary with personality types.

Little if any serious research has been done on cognitive style in second language acquisition. Researchers on "interlanguage" (Richards 1972, Selinker 1972) have suggested that certain individualized strategies may affect language acquisition, but such strategies have not yet been adequately defined or researched.

Using a model proposed by Hill (1972), Baecher (1973) studied the cognitive styles of Spanish-A merican elementary school children in Pontiac, Michigan, in an effort to determine the relationship of cognitive style to linguistic competence in English. The results of Baecher's study were not conclusive, but they did support the hypothesis that a child learning a second language can be taught in various ways depending upon his own affective-cognitive "map."

Some specific research is necessary before teachers and 
materials developers can adequately account for cognitive style. Four specific variables within cognitive style appear to be possible areas of fruitful research in second language acquisition: a) reflective-impulsive thinking, b) broad-narrow categorizing, c) skeletonizing-embroidering, and d) belief congruence-contradiction. Again, only a few of many possible variables are considered here, but in the almost total absence of research on cognitive style, these few variables might indeed provide significant points of departure.

Any observant teacher recognizes basic personality tendencies toward impulsivity on the one hand, and reflectivity on the other. A number of psychological tests are available to determine the degree to which a person tends to make either a quick, or gambling (impulsive) guess at an answer to a problem, or a slower, more calculated (reflective) decision. The implications for language acquisition are numerous. It has been found that children who are conceptually reflective tend to make fewer errors in reading than do conceptually impulsive children (Kagan 1965). On the other hand, impulsive persons may be faster readers. A study conducted at the University of Michigan (Doron 1973) sought to examine the relationship between reflectivity-impulsivity (RI) and reading proficiency in students of English as a second language. Kagan's Matching Familiar Figures test was used to measure RI in a sample of ESL students; Doron then administered reading tests of comprehension and speed to the same subjects to determine the correlation between $\mathrm{RI}$ and reading. She discovered that reflective students were slower and more accurate than impulsive students, and suggested that this fact be taken into account in the teaching of reading in ESL. Further research on RI could indicate kinds of exercises and drills that are suited to both reflective and impulsive personalities. Inductive reasoning was found to be more efficient in reflective children in one study (Kagan, Pearson, and Welch 1966); perhaps the success of inductive teaching methods therefore varies depending upon the RI index of individual students. Future research might indicate a tendency for those with impulsive personalities to go through a number of rapid transitions of semigrammatical stages of interlanguage, with reflective persons tending to remain longer at a particular stage with "larger" leaps from stage to stage.

A cognitive style closely related to $R I$ is the tendency of learners to manifest a consistent preference for either broad or narrow categorization. Narrow categorizers, like impulsive learners, are often more willing to take the risk of being wrong in problemsolving situations, by reason of an overattention to subordinate 
concepts. Evidence of shifting from narrow to broad categorizing is found in first language acquisition in English when children first produce past tense forms of verbs as separate, narrowly categorized items, saying "drank," "caught," and "went"' at one early stage, but then shift to a broad classification of all verbs in a regularized category, saying "drinked," "catched," and "goed." Eventually verbs are subclassified into regular and irregular forms. Similar strategies may play a part in adult second-language acquisition, but since adults tend to be more rigid, and less likely to manifest the same degree of style-shifting, the intra-individual variation may not be quite as prevalent. In general, the relationship of broad and narrow categorization to second language acquisition has not even begun to be researched. Yet if a teacher were aware of such tendencies in an individual, he might be better able to analyze "errors" as the products of consistent cognitive strategies. Materials and textbooks could be designed to compensate for both tendencies by stressing hierarchies of structure. Thus the attention of broad categorizers could be directed to the lower subordinate categories, and narrow categorizers could be encouraged to grasp higher superordinate categories. Grammar teaching in particular could be made more efficient by the use of broad and narrow categories of data.

A third type of cognitive style is the tendency for some individuals to "skeletonize" and others to "embroider" in the recall of cognitive material. The need to simplify the representation and storage of information in cognitive structure is probably universal. But some studies (Holzman and Gardner 1960, Uhlmann and Saltz 1965) support the notion that simplification strategies have both inter-individual and intra-individual variations. In the recall of narrative material, for example, skeletonizing involves "pruning" out some particulars by retaining a substantive core of general facts which subsume the details (Brown 1972a); embroidering, on the other hand, involves "importing," or adding some material in order to retain original details which otherwise might be forgotten. Embroidery is a natural off shoot of the human intellectual tendency toward "closure"; sometimes one will perceive something that is not present in the data simply because he extrapolates beyond the overt stimuli. Carmichael, Hogan and Walter (1932) showed how embroidery can be verbally stimulated; recall of a simple visual line design varied depending upon the verbal stimulus attached to the visual stimulus. Evidence of skeletonization and embroidery is also found in production styles. We have all experienced "yarn spinners" who can conjure up countless exaggerated additions in telling or retelling a story; it is 
also common to find people who provide only the bare facts of an event. Such preferences could play an important role in assessing comprehension as well as production in second language learning. Writing skill might be a particularly fruitful area in which to try to discover how the tendency to embroider or skeletonize relates to style, efficiency, and teachability of writing. Some tests of oral production require a testee to retell a previously read story; the interpretation of the results of such tests could be biased toward embroiderers by sheer quantity of output. While either style can be quite efficient, one tends to judge embroidery as more desirable in some situations and skeletonizing as more appropriate for others. A more careful study of this feature of cognitive style might help to indicate how such preferences may influence actual language acquisition or our judgment of language proficiency.

A fourth category in cognitive style is belief congruence and contradiction. Individual differences exist with respect to the basic need each person feels for internal consistency within his belief system. Some individuals are undoubtedly more content than others to entertain and even internalize contradictory propositions, usually by a process of compartmentalization. Others tend to reject any item which is contradictory or slightly incongruent with their existing system. The notions of "open and closed" mindedness and "dogmatism" are related to the concept of belief congruence. Those who are more dogmatic are inclined to accept only certain highly consistent facts (the closed mind) and usually possess an intolerance for ambiguity as well as a tendency toward premature closure. Hunt (1961) and Ausubel (1949), among others, studied belief congruence extensively, and claimed that both tendencies-a liberal tolerance of contradiction and a high degree of intolerancehave advantages and disadvantages in terms of cognitive efficiency. Second language teachers and researchers need to discover how such differences may affect language learning. Gardner and Lambert (1972) included the categories of dogmatism and authoritarianism in their studies of motivation in second language learning. Ausubel (1968) noted that open-minded persons tend to score higher on tests of verbal ability than do closed-minded individuals; is there a similar correlation in second language learners? More specifically, can methods and materials in second language teaching somehow not be too new and different for the closed-minded individual and still be challenging enough for the open-minded? In order to reach those who are intolerant of ambiguity, either by "nature" or by "nurture," the teacher and materials-developer have the responsibility of offering as clear an explanation of language as possible with a minimum of conflicting evidence. Continued 
ambivalent responses from the teacher to such a student could severely impair his progress in learning the second language.

\section{Conclusions and implications}

A number of categories have been presented here which suggest areas of research and inquiry for the years ahead. If we are to gain a better understanding of language acquisition processes, some, if not all, of the areas discussed here will need to be explored thoroughly. It will be an enormous task. First of all, we are dealing with an extremely complex process within a complex organism; countless variables are interacting and it will be difficult enough to identify even the most salient factors. Second, as more and more variables are found to be influencing the process of second language acquisition, it will become increasingly difficult to type individuals and to classify groups of individuals together. Each person appears to be a unique complex of variables. Third, individuals tend to be somewhat inconsistent within themselves from day to day, or even moment to moment. In a given situation a person may be impulsive, inhibited, and closed-minded, while a change in time or environment may produce a complete reversal. Clearly there are enduring consistencies within individuals, but it is difficult to discover these accurately without systematic observation of a person in a variety of circumstances. Fourth, a source of difficulty in research in second language teaching is found in the teacher-student interaction. In studying "natural" second language acquisition one does not have the imposed, man-made variables such as teachers, textbooks, and other factors that one finds in the educational setting. One always has to consider, for example, how the imposed personality and cognitive style of a teacher will affect a student with his own set of cognitive and affective preferences.

Despite the enormousness of the task, there is good reason to press for solutions to the mysteries of second language acquisition. We have spent many decades hopping from one bandwagon to another, placing almost blind faith in a single method or discipline at one point, then turning to another method, and yet another, always rejecting the previous theory when it broke down or faltered. Now, for perhaps the first time since Gouin wrote his famous L'art d'enseigner et d'étudier les langues (1880), we are attempting to take a genuine interdisciplinary approach to second language research. Neither linguistics nor psychology nor any other discipline alone will produce final answers. The next decade should provide us with information about the human person which, when creatively interrelated with our accumulated knowledge in theoretical 
linguistics, sociolinguistics, psycholinguistics, and the psychology of learning, will possibly enable us to construct a viable theory of second language acquisition.

\section{REFERENCES}

Ausubel, David, P. 1949. Ego development and the learning process. Child Development 20.173-190.

Ausubel, David P. 1952. Ego Development and the Personality Disorders. New York: Grune and Stratton.

Ausuble, David P, 1968. Educational Psychology: A Cognitive View. New York: Holt, Rinehart \& Winston.

Baecher, Richard E. 1973, A pilot study to determine levels of educational development, reading levels, and selected elements of the cognitive styles of Chicago students in the fourth and fifth grades in Pontiac, Michigan. Unpublished Doctoral Dissertation, University of Michigan.

Bandura, Albert and Richard H. Walters. 1963. Social Learning and Personality Development. New York: Holt, Rinehart \& Winston.

Begin, Yves. 1971. Evaluative and Emotional Factors in Learning a Foreign Language. Montreal: Bellarmin.

Brown, H. Douglas. 1972a. Cognitive pruning and second language acquisition. Modern Language Journal 56.218-222.

Brown, H. Douglas. 1972b. The psychological reality of "grammar" in the ESL classroom. TESOL Quarterly 6.263-269.

Carmichael, L., H. P. Hogan and A. A. Walter. 1932. An experimental study of the effect of language on visually perceived form. Journal of Experimental Psychology 15.73-86.

Curran, Charles A. 1972. Counseling-Learning: A Whole-Person Model for Education. New York: Grune and Stratton.

Dollard, J., L. W. Doob, N. E. Miller, O. H. Mowrer and R. R. Sears. 1939. Frustration and Aggression. New Haven, Ct., Yale University Press.

Doron, Sandra. 1973. Reflectivity-impulsivity and their influence on reading for inference for adult students of ESL. Unpublished manuscript, University of Michigan.

Freud, Sigmund. 1920. A General Introduction to Psychoanalysis. New York: Liveright.

Gardner, Robert C. and Wallace E. Lambert. 1972. Attitudes and Motivation in Second Language Learning. Rowley, Ma.: Newbury House.

Gouin, Francois. 1880. L'art d'enseigner et d'étudier les langues. Paris: Fischbacher.

Guiora, Alexander, Benjamin Beit-Hallami, Robert C. I. Brannon, Cecelia Dull and Thomas Scovel. 1970. The effects of experimentally induced changes in ego states on pronunciation ability in a second language: an exploratory study. Unpublished manuscript, University of Michigan.

Guiora, Alexander, Robert C. L. Brannon and Cecelia Dull. 1972. Empathy and second language learning. Language Learning 22.111-130.

Hilgard, Ernest. 1963. Motivation in learning theory. In S. Koch (ed.) Psychology: A Study of Science (Vol. 5.253-283). New York: McGrawHill.

Hill, Joseph E. 1972. The Educational Sciences. Detroit: Oakland Community College. 
Holzman, P. S. and R. W. Gardner. 1960. Leveling-sharpening and memory reorganization. Journal of Abnormal Social Psychology 61.176-180.

Hunt, James McV. 1961. Intelligence and Experience. New York: Ronald Press.

Kagan, Jerome. 1965. Reflection-impulsivity and reading ability in primary grade children. Child Development 36.609-628.

Kagan, Jerome, L. Pearson and Lois Welch. 1966. Conceptual impulsivity and inductive reasoning. Child Development 37.583-594.

Krathwohl, David R., Benjamin Bloom and Bertram B. Masia. 1964. Taxonomy of Educational Objectives, Handbook II: Affective Domain. New York: David McKay Co.

LaForge, Paul. 1971. Community language learning: a pilot study. Language Learning 21.45-61.

Lederer, Elaine. 1973. The effect of motivational factors on the learning ability for second language acquisition. Unpublished manuscript, University of Michigan.

Lukmani, Yasmeen. 1972. Motivation to learn and language proficiency. Language Learning 22.261-274.

McNeill, David. 1966. Developmental psycholinguistics. In F. Smith and G. Miller (eds.) The Genesis of Language (15-84). Cambridge, Ma.: Massachusetts Institute of Technology Press.

Miller, N. E. and J. Dollard. 1941. Social Learning and Imitation. New Haven, Ct.: Yale University Press.

Mowrer, O. Hobart. 1960. Learning Theory and the Symbolic Processes. New York: Wiley.

Nelson, Robert J. and Leon A. Jakobovits. 1970. Motivation in foreign language learning. Northeast Conference on Teaching Foreign Languages 34-104.

Richards, Jack C. 1971. Error analysis and second language strategies. Language Sciences (October) 12-22.

Savignon, Sandra. 1972. Communicative Competence: An Experiment in Foreign Language Teaching. Philadelphia: Center for Curriculum Development.

Selinker, Larry. 1972. Interlanguage. International Review of Applied Linguistics $10.209-231$.

Stevick, Earl. 1973. Review of Curran, Counseling-Learning. A WholePerson Model for Education. Language Learning 23 (in press).

Uhlman, F. W. and E. Saltz. 1965. Retention of anxiety material as a function of cognitive differentiation. Journal of Personal and Social Psychology $1.55-62$. 